Diabetologe 2022 $\cdot 18: 359-367$

https://doi.org/10.1007/s11428-022-00868-7

Angenommen: 4. Februar 2022

Online publiziert: 7. März 2022

(c) The Author(s), under exclusive licence to Springer Medizin Verlag GmbH, ein Teil von Springer Nature 2022

\section{Bedeutung von Glukosewerten im Krankenhaus}

\author{
Michael Jecht \\ Diabetesschwerpunktpraxis, Berlin, Deutschland
}

\section{In diesem Beitrag}

- Blutzuckerzielwerte bei stationärer Behandlung

- Insulintherapie im Krankenhaus

- Blutzuckermonitoring

Am Krankenbett • Kontinuierliche Glukosemessung

- Hypoglykämien im Krankenhaus Schriftlich niedergelegtes Verfahren zum Vorgehen bei Hypoglykämie • Auslösende Ereignisse und Vorbeugung von Hypoglykämie

- Selbstmanagement im Krankenhaus

- Stationäre Behandlung mit Kortisonpräparaten

- Perioperative Versorgung

- Bedeutung präoperativer Blutzuckerwerte

- Orale Antidiabetika im stationären Setting

- Schlussfolgerung

\section{Zusammenfassung}

Hintergrund: Eine Hyperglykämie bei Menschen mit und ohne Diabetes, die ins Krankenhaus eingeliefert werden, ist mit einem erheblichen Anstieg von Morbidität, Mortalität und Gesundheitskosten verbunden. Während eines Krankenhausaufenthaltes treten Stoffwechseldekompensationen häufig als Folge unterschiedlicher Ereignisse oder Zusatztherapien auf. Aufgrund des erhöhten Risikos für eine Zunahme der Morbidität, verbunden mit längerem Krankenhausaufenthalt sowie höheren Kosten und Mortalität, erscheint eine genaue Betrachtung der Bedeutung von Glukosewerten und der Therapieformen im Krankenhaus sinnvoll und angebracht.

Material und Methode: Aktuelle Befunde, Übersichtsarbeiten und Grundlagendaten wurden analysiert und in einer kurzen Übersicht zusammengefasst und diskutiert.

Fazit: Eine persistierende Hyperglykämie im Krankenhaus ist häufig und oft mit unzureichenden Ergebnissen des Krankenhausaufenthaltes verbunden. Die kontinuierliche Insulininfusion bleibt die Therapie der Wahl während hyperglykämischer Krisen und kritischen Erkrankungen. Auch bei nicht kritisch kranken Menschen mit ausgeprägter Hyperglykämie, schon ambulant bekannten hohen Insulindosen, mit Typ-1-Diabetes oder mit steroidinduzierter Hyperglykämie bleibt Insulin das Mittel der Wahl.

\section{Schlüsselwörter}

Diabetes mellitus · Hyperglykämie · Hypoglykämie · Insulin · Krankenhausaufenthalt

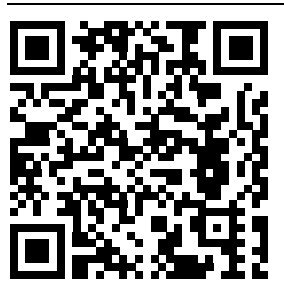

QR-Code scannen \& Beitrag online lesen
Betrachtet man den hohen Anteil an Diabetespatienten in deutschen Krankenhäusern, kann man davon ausgehen, dass die Anzahl der stationär behandelten Menschen mit Diabetes unterschätzt wird. Unberücksichtigt bleibt bei diesen Statistiken, dass bei Menschen mit Diabetes im Vergleich zu solchen ohne diese Stoffwechselerkrankung für dieselbe Hauptdiagnose eine längere Krankenhausaufenthaltsdauer, mehr Komplikationen und eine höhere Mortalitätsrate zu verzeichnen sind. In den Krankenhausstatistiken werden in der Regel nur die Hauptdiagnosen, die zur Aufnahme führten, berücksichtigt. Der Diabetes selbst dagegen wird im DRG-System (DRG "diagnosis related groups") nur als Begleiterkrankung (sog. Nebendiagnose) dokumentiert [1].

Eine Hyperglykämie bei Menschen mit und ohne Diabetes, die ins Krankenhaus eingeliefert werden, ist mit einem erheblichen Anstieg von Morbidität, Mortalität und Gesundheitskosten verbunden [2-4]. Insbesondere sind der Diabetes mellitus und eine durch (operativen) Stress bedingte Hyperglykämie häufig anzutreffen, verbunden damit sind ein Anstieg der Krankenhauskomplikationen, der Aufenthaltsdauer und der Sterblichkeit [2-7]. Auch treten während eines Krankenhausaufenthaltes Stoffwechseldekompensationen häufig als Folge einer elektiven operativen Maßnahme auf, aber auch durch Therapien während des stationären Aufenthaltes - mit Psychopharmaka, Onkologika oder Steroiden [3, 4].

Dementsprechend sind adäquate Diabetestherapien zwingend notwendig, um eine optimale Blutzuckereinstellung zu erreichen und damit Komplikationen zu vermeiden $[1,5]$. Weiterhin muss betont wer- 
den, dass eine strukturierte interdisziplinäre Zusammenarbeit in einer Klinik oder einem Klinikverbund eine effektivere Diabetesbetreuung zur Folge hat und die Liegedauer reduziert [8].

Darüber hinaus zeigten Daten aus der COVID-19-Pandemie (COVID-19 "coronavirus disease $2019^{\prime \prime}$ ), wie gefährdet Menschen mit Typ-1- und Typ-2-Diabetes im Vergleich zu Patienten ohne diese Stoffwechselerkrankung für die Entwicklung von Komplikationen im Krankenhaus sind $[9,10]$.

\section{॥) Während des stationären Aufenthaltes ist eine adäquate Diabetestherapie unverzichtbar}

Wie bei der Hyperglykämie ist auch die stationäre Hypoglykämie mit schlechten Ergebnissen und erhöhten Gesundheitskosten verbunden. Diese Assoziation könnte die Schwere der Erkrankung und höhere Raten von Komorbiditäten bei Menschen widerspiegeln, die eine Hypoglykämie entwickeln [11].

Die Zahl der Patienten in deutschen Krankenhäusern mit einem Diabetes mellitus (Nebendiagnose) wurde in einer kürzlich veröffentlichten Arbeit dargestellt. Das Ergebnis dieser Untersuchung basiert auf einer Erhebung aus den Jahren 2015-2017 und wurde mit Hilfe des DRGSystems bei vollstationär behandelten Patienten, die ein Lebensalter von 20 oder höher aufwiesen, erhoben: Etwa 18\% von ca. 16,5 Mio. stationären Fälle hatten eine Haupt- oder Nebendiagnose Diabetes mellitus (2017: Typ-2-Diabetes 17,1\%; Typ-1-Diabetes 0,5\%). Diese Erkrankung war bei den männlichen stationären Fällen häufiger als bei den weiblichen (2017: Typ-2-Diabetes $19,7 \%$ vs. $14,8 \%$, Typ-1Diabetes $0,5 \%$ vs. 0,4\%). Im Vergleich zu den Fällen ohne Diabetes bestand 2017 der größte Unterschied in der Verweildauer bei Patienten mit einem Typ-1-Diabetes im Alter von 40-49 Jahren (7,3 vs. 4,5 Tage) und in der Krankenhaussterblichkeit bei Menschen mit Typ-2-Diabetes im Alter von $70-79$ Jahren (3,7\% vs. 2,8\%; [12]).

Verschiedene internationale Fachgesellschaften empfehlen die Insulintherapie als Eckpfeiler des pharmakologischen Managements im Krankenhaus. Die i.v. Insulintherapie ist dabei in der Intensiv- behandlung die Maßnahme der Wahl. Die „American Diabetes Association“ (ADA) erstellt bekanntermaßen regelmäßig eine aktualisierte Reihe von Empfehlungen, die mehrere Aspekte der stationären Diabetesversorgung [13] berücksichtigen - im vorliegenden Beitrag wurden unterschiedliche Teilaspekte daraus übernommen.

In Großbritannien erarbeiteten die „Joint British Diabetes Societies“ (JBDS) eine Reihe von Leitlinien zu verschiedenen Aspekten der stationären Versorgung für bestimmte Bevölkerungsgruppen, einschließlich der jüngsten Empfehlungen zu COVID-19 [14].

Die „Endocrine Society" veröffentlichte in Zusammenarbeit mit anderen Gesellschaften 2012 Leitlinien für das Management von Diabetes und Hyperglykämie in Krankenhauseinheiten ohne entsprechende Intensivstationsbehandlung [7].

Da die Evidenz oft unzureichend ist, um zu bestimmen, wie die verschiedenen Aspekte der stationären Therapie am besten zu handhaben sind, wohnt diesen Leitlinien oft ein großes Element der Konsensmedizin inne.

Die Deutsche Diabetes Gesellschaft hat bisher keine entsprechende Übersicht zur Behandlung des Diabetes mellitus im Krankenhaus herausgegeben, sondern nur Maßnahmen für den stationären Bereich im Rahmen einer COVID-19-Erkrankung dargelegt.

Betrachtet man die Standards der ADA zur Diabetesversorgung im Krankenhaus, lassen sich die im Folgenden dargestellten Gesichtspunkte herleiten [13]:

\section{Blutzuckerzielwerte bei stationärer Behandlung}

Eine Insulintherapie sollte zur Behandlung einer persistierenden Hyperglykämie $a b$ einem Schwellenwert von $\geq 180 \mathrm{mg} / \mathrm{dl}$ $(10,0 \mathrm{mmol} / \mathrm{l})$ eingeleitet werden. Sobald die Insulintherapie begonnen wurde, wird für die meisten kritisch sowie nicht kritisch kranken Patienten ein Blutzuckerzielbereich von $140-180 \mathrm{mg} / \mathrm{dl}$ (7,8-10,0 mmol/l) empfohlen.

Noch strengere Ziele, wie $110-140 \mathrm{mg} / \mathrm{dl}$ (6,1-7,8 mmol/l), können für ausgewählte Patienten angemessen sein, wenn sie ohne signifikante Hypoglykämie erreicht werden können.
Grundsätzlich wird von einer Hyperglykämie bei hospitalisierten Patienten gesprochen, wenn der Blutzuckerspiegel über $140 \mathrm{mg} / \mathrm{dl}(7,8 \mathrm{mmol} / \mathrm{l})$ liegt $[7,15$, $16]$, und es sollten dann entsprechende Maßnahmen umgesetzt werden, wie z.B. eine Umstellung der Ernährung oder eine Änderung der Medikamente, die eine Hyperglykämie verursachen. Ein $\mathrm{HbA}_{1 c^{-}}$ Wert $\left(H b A_{1 c}\right.$ glykiertes Hämoglobin) bei einer Krankenhausaufnahme von $\geq 6,5 \%$ $(48 \mathrm{mmol} / \mathrm{mol})$ deutet bekanntermaßen darauf hin, dass der Beginn des Diabetes vor dem Krankenhausaufenthalt lag.

Bezüglich der Einschätzung erhöhter Blutzuckerwerte sind v. a. 2 Arbeiten zu nennen: Van den Berghe et al. [17] wiesen nach, dass ein intensives i.v. Insulinregime zum Erreichen eines Blutzuckerzielbereichs von $80-110 \mathrm{mg} / \mathrm{dl}(4,4-6,1 \mathrm{mmol} / \mathrm{l})$ die Sterblichkeit um $40 \%$ reduzierte, verglichen mit einem Standardansatz, bei welchem ein Blutzuckerspiegel von $180-215 \mathrm{mg} / \mathrm{dl}$ (10-12 mmol/l) bei kritisch kranken Patienten mit kürzlich erfolgter Operation angestrebt wurde. Diese Studie lieferte handfeste Beweise dafür, dass eine aktive Behandlung zur Senkung des Blutzuckerspiegels bei hospitalisierten Patienten unmittelbare Vorteile mit sich bringt.

\section{I) Moderate Blutzuckerzielwerte sind zu bevorzugen}

Die Autoren einer großen, multizentrischen Folgestudie, der sog. NICE-SUGARStudie (NICE-SUGAR "normoglycemia in intensive care evaluation and survival using glucose algorithm regulation $^{\prime \prime}$ ), kamen jedoch zu einem anderen Schluss, sodass der optimale Zielbereich für die Blutzuckerspiegelsenkung bei kritischen Erkrankungen neu überdacht wurde [18]: In dieser Studie hatten kritisch kranke Patienten, die auf eine intensive Blutzuckerkontrolle (80-110 mg/dl) randomisiert wurden, keinen signifikanten Behandlungsvorteil gegenüber einer Gruppe mit moderateren Blutzuckerzielen (140-180 mg/dl, [7,8-10,0 mmol/l]) und wiesen sogar eine leicht, aber signifikant höhere Sterblichkeit auf ( $27,5 \%$ vs. $25 \%$ ). Bei der intensiv behandelten Gruppe wurde eine 10- bis 15-fach höhere Hypoglykämierate beobachtet, was zu den 
Hier steht eine Anzeige.

黑 Springer 
festgestellten ungünstigen Ergebnissen beigetragen haben könnte. Die Ergebnisse von NICE-SUGAR werden durch die Resultate mehrerer Metaanalysen gestützt, von denen einige ebenfalls darauf hindeuten, dass eine strenge Kontrolle des Blutzuckerspiegels die Sterblichkeit im Vergleich zu moderateren Blutzuckerzielwerten erhöht und im Allgemeinen auch zu höheren Hypoglykämieraten führt [19-21].

Es lässt sich also festhalten, dass eine Insulintherapie zur Behandlung einer persistierenden Hyperglykämie von $\geq 180 \mathrm{mg} / \mathrm{dl}$ (10,0 $\mathrm{mmol} / \mathrm{l})$ eingeleitet und bei der Mehrzahl der kritisch kranken Patienten auf einen Glukosebereich von $140-180 \mathrm{mg} / \mathrm{dl}(7,8-10,0 \mathrm{mmol} / \mathrm{l})$ ausgerichtet werden sollte.

Blutzuckerziele, wie $110-140 \mathrm{mg} / \mathrm{dl}$ (6,1-7,8 mmol/l), können für ausgewählte Patienten (z.B. kritisch kranke Menschen postoperativ oder Patienten mit Herzoperationen) angemessen sein, solange sie ohne signifikante Hypoglykämie erreicht werden [22, 23].

Andererseits können Glukosekonzentrationen zwischen 180 und $250 \mathrm{mg} / \mathrm{dl}$ (10 und $13,9 \mathrm{mmol} / \mathrm{l}$ ) bei Patienten mit schweren Begleiterkrankungen und in stationären Einrichtungen akzeptabel sein, in denen eine häufige Überwachung des Blutzuckerspiegels oder eine engmaschige pflegerische Betreuung nicht möglich sind. Bei todkranken Patienten mit kurzer Lebenserwartung sollten Werte von $250 \mathrm{mg} / \mathrm{dl}(13,9 \mathrm{mmol} / \mathrm{l})$ als ausreichend angesehen werden. Bei ihnen sind weniger aggressive Insulinschemata zur Minimierung von Glukosurie, Dehydratation und Elektrolytstörungen oft angemessener [24].

\section{Insulintherapie im Krankenhaus}

Meistens wird in klinischen Leitlinien empfohlen, orale Antidiabetika während des Krankenhausaufenthaltes abzusetzen. Dennoch wird bei einigen Patienten mit Typ-2-Diabetes die Behandlung mit oralen Antidiabetika fortgesetzt, sodass es sinnvoll erscheint, die unterschiedlichen Aspekte differenzierter zu betrachten. Dabei ergeben sich die im Folgenden dargestellten Gesichtspunkte, die in der Regel für eine Insulintherapie sprechen. Eine Fortsetzung der Behandlung mit oralen Antidiabetika muss insgesamt immer kritisch hinterfragt werden, da eine Niereninsuffizienz oder Verschlechterung der Leberfunktion akut auftreten können.

Ergebnisse aus randomisierten Studien zeigten bei auf einer Normalstation im Krankenhaus behandelten Patienten mit Typ-2-Diabetes durchweg eine bessere Blutzuckereinstellung unter einer intensivierten Insulintherapie (ICT intensivierte konventionelle Insulintherapie) als unter einer Behandlung nur mit einem Insulinschema mittels fest vorgegebener Dosen bezüglich bestimmter Blutzuckerintervalle $[25,26]$.

Um Hypoglykämien zu vermeiden, wird oft bei Patienten mit Diabetes, die nüchtern sind oder bei denen operative oder diagnostische Eingriffe erforderlich sind, ein sog. Basal-Plus-Ansatz empfohlen, d. h. die Gabe eines Basalinsulins und ggf. Korrekturen mit einem schnell wirkenden Insulin.

\section{I) Auf Intensivstationen ist die i.v. Insulingabe die Therapie der Wahl}

Auf einer Intensivstation ist die kontinuierliche Insulininfusionstherapie die bevorzugte Behandlung für Intensivpatienten mit Hyperglykämie, einschließlich solcher ohne Diabetesdiagnose sowie für die meisten Patienten mit hyperglykämischen Krisen [5, 14, 27, 28]. Zusätzlich können auch Menschen mit schwerer, durch Steroide induzierter Hyperglykämie oder solche, die sich einer Transplantation unterziehen, von einer kontinuierlichen Insulininfusion profitieren.

\section{Blutzuckermonitoring}

\section{Am Krankenbett}

Bei stationär behandelten Patienten mit Diabetes, die essen können und dürfen, sollte die Blutzuckerspiegelkontrolle am Krankenbett vor den Mahlzeiten durchgeführt werden. Bei Menschen, die keine Nahrung zu sich nehmen können bzw. dürfen, wird die Kontrolle des Blutglukosespiegels alle 4-6h empfohlen [15].

Erfolgt eine i.v. Insulintherapie, sollten die Blutzuckerwerte regelmäßig alle 30 min bis alle $2 \mathrm{~h}$ bestimmt werden. Natürlich müssen dabei zwingend Sicherheitsstandards eingehalten werden, die die ge- meinsame Nutzung von Lanzetten sowie anderen Testmaterialien und Nadeln verbieten [28].

Die überwiegende Mehrheit der Blutzuckermessungen in Krankenhäusern wird mit Standardglukosemessgeräten und Kapillarblut durchgeführt, das mit "fingersticks" entnommen wird, ähnlich wie bei der ambulanten Blutzuckermessung zu Hause [29].

Dabei sind bekanntermaßen die sog. POC-Messgeräte ( $P O C$ "point of care") nicht so genau wie die Messgeräte im Labor, außerdem können die Blutzuckermessungen aus Kapillarblut aufgrund von Perfusion, Ödemen, Anämie, Erythrozytose und bedingt durch verschiedene, im Krankenhaus verwendete Medikamente Artefakte aufweisen [30]. Von entscheidender Bedeutung ist jedoch, dass die für den Einsatz im Krankenhaus ausgewählten Geräte und die Arbeitsabläufe, in denen sie verwendet werden, einer sorgfältigen Leistungs- und Zuverlässigkeitsanalyse sowie einer laufenden Qualitätsbewertung unterzogen werden.

\section{Kontinuierliche Glukosemessung}

Die kontinuierliche Glukosemessung (CGM) ermöglicht häufige Messungen des interstitiellen Glukosespiegels sowie der Richtung und des Ausmaßes der Glukosetrends. Obwohl die CGM theoretische Vorteile gegenüber der POC-Glukosemessung bei der Erkennung und Verringerung der Häufigkeit von Hypoglykämien hat, wurde sie bisher von der FDA („U.S. Food and Drug Administration") nicht für die stationäre Anwendung zugelassen. Einige Krankenhäuser mit etablierten Glukosemanagementteams erlauben den Einsatz von CGM bei ausgewählten Patienten auf individueller Basis, sofern sowohl die Patienten als auch das Glukosemanagementteam in der Anwendung dieser Technologie gut geschult sind. Die CGM stellt bei hochinfektiösen Patienten, die z. B. eine COVID-19-Erkrankung haben und beatmet sind, eine mögliche Alternative $\mathrm{da}$, dennoch ist dieses Verfahren bisher auf Intensivstationen nicht zugelassen. 


\section{Hypoglykämien im Krankenhaus}

\section{Schriftlich niedergelegtes Verfahren zum Vorgehen bei Hypoglykämie}

Jedes Krankenhaus oder Krankenhaussystem sollte über ein Verfahren verfügen und dieses schriftlich niedergelegt haben, in dem der sofortige Umgang mit Unterzuckerungen beschrieben ist und nach dem weitere Maßnahmen zur Analyse umzusetzen sind. Stattgefundene Hypoglykämien während eines Krankenhausaufenthaltes sollten in der Krankenakte dokumentiert und nachverfolgt werden [16]. Zudem sollte das Behandlungsschema der hiervon Betroffenen regelmäßig überprüft und ggf. geändert werden, um weitere Hypoglykämien zu verhindern, wenn ein Blutzuckerwert von $<70 \mathrm{mg} / \mathrm{dl}(3,9 \mathrm{mmol} / \mathrm{l})$ aufgetreten ist.

\section{॥ Es sollten individuelle Pläne zur Prävention und Behandlung von Hypoglykämien erstellt werden}

Darüber hinaus sollten individuelle Pläne zur Prävention und Behandlung von $\mathrm{Hy}$ poglykämien für jeden Patienten entwickelt werden. In einer Konsenserklärung der ADA und der AACE („American Association of Clinical Endocrinologists") wird empfohlen, das Behandlungsschema eines Patienten zu überprüfen, sobald ein Blutzuckerspiegel von $<70 \mathrm{mg} / \mathrm{dl}(3,9 \mathrm{mmol} / \mathrm{l})$ auftritt, da solche Werte häufig eine nachfolgende schwere Hypoglykämie vorhersagen [15].

\section{Auslösende Ereignisse und Vorbeugung von Hypoglykämie}

Insulin ist eines der häufigsten Medikamente, die bei Krankenhauspatienten unerwünschte Ereignisse verursachen, bedingt meistens durch Fehler bei der Insulindosierung und/oder -verabreichung. Abgesehen von Insulindosierungsfehlern sind häufige vermeidbare Ursachen bedingt durch:

- eine unsachgemäße Verschreibung anderer glukosespiegelsenkender Medikamente,

- die unsachgemäße Behandlung der ersten Hypoglykämieepisode und
- ein Missverhältnis zwischen Ernährung und Insulin, das häufig auf eine unerwartete Unterbrechung der Nahrungsaufnahme zurückzuführen ist [31-33].

Weiterhin ist eine akute Niereninsuffizienz als ein wichtiger Risikofaktor für eine $\mathrm{Hy}$ poglykämie im Krankenhaus zu beachten, möglicherweise als Folge einer verminderten Insulinausscheidung [34].

Neben Fehlern bei der Insulinbehandlung kann eine iatrogene Hypoglykämie ausgelöst werden durch

- eine plötzliche Verringerung der Kortikosteroiddosis,

- eine verringerte orale Nahrungsaufnahme,

- Erbrechen,

- einen ungeeigneten Zeitpunkt für die Verabreichung von kurz wirksamen Insulinen im Verhältnis zu den Mahlzeiten,

- eine verringerte Infusionsrate von i.v. Traubenzuckerlösung,

- eine unerwartete Unterbrechung der enteralen oder parenteralen Ernährung,

- verspätete oder versäumte Blutzuckerspiegelkontrollen und

- eine veränderte Fähigkeit des Patienten, Symptome zu melden [35].

\section{Selbstmanagement im Krankenhaus}

Das Selbstmanagement eines Diabetes im Krankenhaus kann für bestimmte $\mathrm{Pa}$ tienten geeignet sein $[36,37]$ und sollte grundsätzlich auch stets ermöglicht werden. In Frage kommen sowohl jugendliche als auch erwachsene Patienten, die ihre Diabetestherapie und Blutzuckerspiegeleinstellung zu Hause erfolgreich durchführen und deren kognitive und körperliche Fähigkeiten, die für die erfolgreiche Selbstverabreichung von Insulin und die Selbstkontrolle des Blutglukosespiegels erforderlich sind, während des Krankenhausaufenthaltes durch neu aufgetretene Erkrankungen nicht beeinträchtigt sind.

Darüber hinaus sollten sie Kohlenhydrate gut einschätzen können, mehrmals täglich Insulin spritzen oder eine kontinuierliche subkutane Insulininfusion (CSII) durchführen, einen stabilen Insulinbedarf haben und den Umgang mit Erkrankungssituationen verstehen. Wenn ein Selbstmanagement durch den Patienten durchgeführt werden kann, sollte aber ein Protokoll die entsprechenden Anforderungen und Zustimmungen durch den Patienten und das diesen betreuende Krankenhauspersonal auch aus rechtlichen Gründen enthalten.

Soll eine Insulinpumpentherapie mit oder ohne kontinuierliche Glukosemessung verwendet werden, sollten diese Maßnahmen in den entsprechenden Krankenhausrichtlinien und -verfahren beschrieben sein, in denen die Richtlinien für die Insulinpumpentherapie, einschließlich des Wechsels der Infusionsstellen, festgelegt sind $[38,39]$.

\section{Stationäre Behandlung mit Kortisonpräparaten}

Die Prävalenz einer stationären Therapie mit Kortison beträgt bis zu $10 \%$. Diese Medikamente können bei Patienten mit und ohne Vorerkrankung eine Hyperglykämie auslösen [40]. Glukokortikoidtyp und Wirkungsdauer müssen daher bei der Festlegung von Insulinbehandlungsschemata unbedingt berücksichtigt werden. Täglich eingenommene, kurz wirkende Glukokortikoide wie Prednisolon erreichen ihren Plasmaspitzenwert innerhalb von 4-6h [41], haben aber pharmakologische Effekte, die den ganzen Tag über anhalten.

Bei Patienten, die morgens Steroide einnehmen, kommt es im Laufe des Tages zu einer überproportionalen Hyperglykämie, doch erreichen sie unabhängig von der Behandlung über Nacht häufig normale Blutzuckerwerte [40].

\section{Perioperative Versorgung}

Vielen Standards für die perioperative Versorgung liegt keine solide Evidenz zugrunde. In den Standards der amerikanischen Diabetesgesellschaft für stationäre Patienten ist der folgende Ansatz [13, 42-44] beschrieben:

1. Der Zielbereich für den Blutzuckerspiegel in der perioperativen Phase sollte $80-180 \mathrm{mg} / \mathrm{dl}(4,4-10,0 \mathrm{mmol} / \mathrm{l})$ betragen. 
2. Bei Patienten mit Diabetes mit einem hohen Risiko für eine ischämische Herzerkrankung sowie bei Menschen mit autonomer Neuropathie oder Niereninsuffizienz sollte eine präoperative Risikobewertung durchgeführt werden.

3. Metformin sollte am Tag der Operation abgesetzt werden - besser aber schon 2 Tage prä- und zusätzlich 2 Tage postoperativ.

4. Eine SGLT-2-Inhibitor-Therapie (SGLT-2 "sodium glucose linked transporter 2") muss 3-4 Tage vor der Operation abgesetzt werden.

5. Alle anderen oralen Antidiabetika sollten am Morgen des Tages der Operation oder des Eingriffs abgesetzt werden, und es sollten die Hälfte der $\mathrm{NPH}$-Dosis (NPH neutrales Protamin Hagedorn) oder eine 75 - bis $80 \%$ ige Dosis eines lang wirksamen Analoginsulins oder Pumpenbasalinsulins verabreicht werden.

6. Die Überwachung des Blutzuckerspiegels muss mindestens alle $2-4 \mathrm{~h}$ erfolgen, während der Patient keine Nahrung zu sich nehmen darf, und je nach Bedarf sollte ein kurz wirksames Insulin verwendet werden.

7. Es gibt keine Daten über die Verwendung und/oder den Einfluss von GLP1-Rezeptor-Agonisten (GLP-1 „glucagon-like peptide $1^{\prime \prime}$ ) oder ultralang wirkenden Insulinanaloga auf den Blutzuckerspiegel in der perioperativen Versorgung.

Die Autoren einer Metaanalyse der Cochrane-Initiative kamen zu dem Schluss, dass ein strengerer perioperativer Blutzuckerzielbereich als $80-180 \mathrm{mg} / \mathrm{dl}(4,4-$ $10,0 \mathrm{mmol} / \mathrm{l})$ die Ergebnisse nicht verbessert und mit mehr Hypoglykämien verbunden ist [43]; daher wird im Allgemeinen von strengeren Blutzuckerzielwerten abgeraten.

\section{Bedeutung präoperativer Blutzuckerwerte}

Hinterfragt man die Bedeutung eines präoperativen Blutzuckerwerts, kann man auch eine kürzlich erschiene Arbeit zur Diskussion hinzuziehen [45], in welcher nachgewiesen wurde, dass ein höherer präoperativer Blutzuckerwert signifikant mit einer verlängerten Verweildauer im Krankenhaus assoziiert ist. Eine positive Dosis-Wirkungs-Kurve zwischen präoperativen Blutzuckerwerten und dem bereinigten Risiko einer Verweildauer von über 3 Tagen wurde beschrieben.

Betrachtet man die Studienlage, finden sich immer mehr Hinweise auf den prognostischen Wert des Managements einer präoperativen Hyperglykämie bei Patienten mit diagnostiziertem Diabetes oder bei älteren Erwachsenen, die sich einer elektiven Operation unterziehen [46]. Dennoch gibt es keinen Konsens über ein routinemäßiges Screening des präoperativen Blutzuckerspiegels, da die Evidenz für die Wirksamkeit eines bestimmten Blutzuckerzielbereichs spärlich ist [46-50]. Basierend auf diesen Ergebnissen werden in den neuesten Richtlinien der „European Society of Anaesthesiology" (ESA) keine routinemäßigen Bewertungen von präoperativen Blutzuckerwerten bei Personen, die sich einer elektiven nichtkardialen Operation unterziehen, empfohlen [51].

\section{Orale Antidiabetika im stationären Setting}

Trotz unzureichender Evidenz aus klinischen Studien werden Metformin und andere orale Antidiabetika im Krankenhaus bei Menschen mit Typ-2-Diabetes häufig eingesetzt $[52,53]$. Bei Patienten, bei denen ein Risiko für eine Laktatazidose besteht, wie z. B. bei anaerobem Stoffwechsel (d.h. Sepsis, Hypoxie), eingeschränkter Clearance von Metformin (erhebliche Niereninsuffizienz) oder Milchsäure (Leberversagen), sollte Metformin vermieden werden [54].

Von der Verwendung von Sulfonylharnstoffen wird wegen der Gefahr von Hypoglykämien durch die Fachgesellschaften abgeraten. Eine Ausnahme bilden die britischen Empfehlungen, in denen nahegelegt wird, dass Sulfonylharnstoffe bei der Behandlung einer glukokortikoidinduzierten Hyperglykämie nützlich sein könnten [41].

Für Patienten mit Typ-2-Diabetes und Herzinsuffizienz oder diabetischer Nierenerkrankung sind SGLT-2-Inhibitoren derzeit die glukosespiegelsenkenden Medikamente der Wahl. Studien, in denen ihr
Einsatz während des stationären Aufenthaltes genauer untersucht wurde, stehen noch aus, grundsätzlich jedoch muss festgehalten werden, dass die Anwendung von SGLT-2-Inhibitoren mit einer Reduktion des kombinierten Endpunkts, einer Verschlechterung der Herzinsuffizienz, einer erneuten Hospitalisierung wegen Herzinsuffizienz oder des Todes nach 60 Tagen verbunden ist.

\section{》) Von der Verwendung von Sulfonylharnstoffen wird wegen der Gefahr von Hypoglykämien abgeraten}

Ergebnisse aus mehreren randomisierten kontrollierten Studien und Beobachtungsstudien im Krankenhausumfeld ergaben, dass DPP-4-Inhibitoren (DPP Dipeptidylpeptidase) gut verträglich und wirksam für die Blutzuckerspiegelkontrolle sind, mit einem geringen Risiko für Hypoglykämien bei Patienten mit leichter bis mäßiger Hyperglykämie [55, 56].

Zwar wurden GLP-1-Rezeptor-Agonisten im stationären Bereich getestet [57, 58], jedoch sollte beachtet werden, dass sie mit einer erhöhten Häufigkeit gastrointestinaler Nebenwirkungen einhergehen, was bei der Fortführung der Therapie unter stationären Bedingungen unbedingt beachtet werden muss.

\section{Schlussfolgerung}

Eine persistierende Hyperglykämie im Krankenhaus ist häufig und oft mit unzureichenden Ergebnissen des Krankenhausaufenthaltes verbunden. Die kontinuierliche Insulininfusion bleibt die Therapie der Wahl während hyperglykämischer Krisen und kritischen Erkrankungen.

Bei nicht kritisch Kranken ist Insulin das Mittel der Wahl bei Patienten mit ausgeprägter Hyperglykämie, bei schon ambulant bekannten hohen Insulindosen, bei Typ-1-Diabetes oder bei Menschen mit steroidinduzierter Hyperglykämie.

Bei Patienten mit leichter bis mäßiger Hyperglykämie könnte die Verwendung eines Basal-Plus-Ansatzes mit oder ohne nichtinsulinäre Wirkstoffe die Behandlung (weniger Insulin, weniger Injektionen und weniger Hypoglykämien bei Patienten mit niedrigeren Blutzuckerkonzentratio- 
nen) im Vergleich zu komplexen Insulinregimen, die häufig mit iatrogenen Hypoglykämien einhergehen, vereinfachen.

Der Einsatz von Diabetestechnologien im Krankenhaus entwickelt sich zunehmend weiter, ist aber für eine breite Umsetzung noch nicht geeignet. Die Erfahrungen mit der Nutzung derartiger Technologien im Krankenhausumfeld während der COVID-19-Pandemie werden von unschätzbarem Wert sein. Forschung und politische Veränderungen, die die Integration von Diabetestechnologien in elektronische Krankenakten erleichtern, sind dringend erforderlich. Die Gesundheitssysteme sollten die stationäre Versorgung von Diabetespatienten und Blutzuckerspiegeleinstellungen weiter anpassen und ggf. umgestalten, um eine kosteneffiziente und patientenzentrierte Qualitätsversorgung zu gewährleisten.

\section{Fazit für die Praxis}

- Die Insulintherapie stellt den Eckpfeiler des pharmakologischen Managements im Krankenhaus dar und sollte zur Behandlung einer persistierenden Hyperglykämie ab einem Schwellenwert von $\geq 180 \mathrm{mg} / \mathrm{dl}$ $(10,0 \mathrm{mmol} / \mathrm{l})$ eingeleitet werden.

- Für die meisten kritisch kranken und nicht kritisch kranken Patienten wird ein Blutzuckerzielbereich von $140-180 \mathrm{mg} / \mathrm{dl}$ $(7,8-10,0 \mathrm{mmol} / \mathrm{l})$ empfohlen.

- Die i.v. Insulintherapie ist in der Intensivbehandlung die Maßnahme der Wahl.

- Die Blutzuckerspiegelkontrolle stationär aufgenommener Diabetespatienten, die essen können, sollte am Krankenbett vor den Mahlzeiten durchgeführt werden.

- Bei Patienten, die nicht essen können bzw. dürfen, sollte eine Blutzuckerkontrolle alle 4-6h, bei einer i.v. Insulintherapie alle 30 bis zu 120 min durchgeführt werden.

- Jedes Krankenhaus sollte über ein Verfahren verfügen, in dem der sofortige Umgang mit Unterzuckerungen beschrieben ist.

- Das Selbstmanagement eines Diabetes im Krankenhaus sollte grundsätzlich immer ermöglicht werden.
Hier steht eine Anzeige.

Springer 


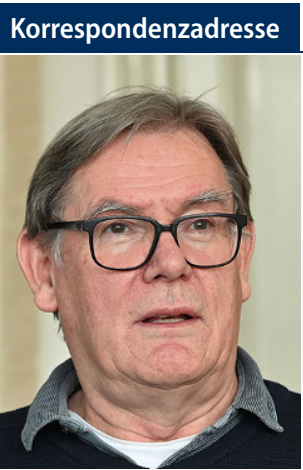

Dr. Michael Jecht

Diabetesschwerpunktpraxis

Rodensteinstr. 32, 13593 Berlin, Deutschland mjecht@t-online.de

\section{Einhaltung ethischer Richtlinien}

Interessenkonflikt. M. Jecht gibt an, dass kein Interessenkonflikt besteht.

Für diesen Beitrag wurden vom Autor keine Studien an Menschen oder Tieren durchgeführt. Für die aufgeführten Studien gelten die jeweils dort angegebenen ethischen Richtlinien.

\section{Literatur}

1. Fritsche A (2017) Diabetes mellitus in der Klinik: Mehr Strukturen schaffen. Dtsch Arztebl 114(41): 16

2. Kufeldt J, Kovarova M, Adolph $M$ et al (2018) Prevalence and distribution of diabetes mellitus in a maximum care hospital: urgent need for HbA1c-Screening. Exp Clin Endocrinol Diabetes 126:123-129

3. Levy N, Dhatariya K (2019) Pre-operative optimisation of the surgical patient with diagnosed and undiagnosed diabetes: a practical review. Anaesthesia 74(Suppl 1):58-66

4. Nationale Diabetes-Surveillance am Robert-KochInstitut (2019) Diabetes in Deutschland - Bericht der Nationalen Diabetes-Surveillance

5. Moghissi ES, Korytkowski MT, DiNardo M et al (2009) American Association of Clinical Endocrinologists and American Diabetes Association consensus statement on inpatient glycemic control. Diabetes Care 32:1119-1131

6. Dhatariya K, Mustafa OG, Rayman G (2020) Safe care for people with diabetes in hospital. Clin Med 20:21-27

7. Umpierrez GE, Hellman R, Korytkowski MT et al (2012) Management of hyperglycemia in hospitalized patients in non-critical care setting: an Endocrine Society clinical practice guideline. JClin Endocrinol Metab 97:16-38

8. Röhling M, Redaelli M, Simic D, Lorrek K, Samel C, Schneider P, Kempf K, Stock S, Martin S (2019) TeDia-A telemedicine-based treatment model for inpatient and interprofessional diabetes care. Diabetes Metab Syndr Obes 12:2479-2487

9. Barron E, Bakhai C, Kar Petal (2020) Associations of type 1 and type 2 diabetes with COVID-19-related mortality in England: a whole-population study. Lancet Diabetes Endocrinol 8:813-822

10. Holman N, Knighton P, Kar Pet al (2020) Riskfactors for COVID-19-related mortality in people with type 1 and type 2 diabetes in England: a populationbased cohort study. Lancet Diabetes Endocrinol 8:823-833

11. Boucai L, Southern WN, Zonszein J (2011) Hypoglycemia-associated mortality is not drugassociated but linked to comorbidities. Am J Med 124:1028-1035

12. Auzanneau M, Fritsche A, Icks A, Siegel E, Kilian R, Karges W, Lanzinger S, Holl RW (2021) Diabetes in the hospital-a nationwide analysis of all hospitalized cases in Germany with and without diabetes, 2015-2017. Dtsch Arztebl Int 118:407-412

13. American Diabetes Association (2021) 15. Diabetes care in the hospital. Standards of medical care in diabetes-2021. Diabetes Care 44(Suppl 1):S211-S220

14. Joint British Diabetes Societies for Inpatient Care group https://abcd.care/joint-british-diabetessocieties-jbds-inpatient-care-group. Zugegriffen: 2. Jan. 2021

15. Moghissi ES, Korytkowski MT, DiNardo $M$ et al (2009) American Association of Clinical Endocrinologists; American Diabetes Association. American Association of Clinical Endocrinologists and American Diabetes Association consensus statement on inpatient glycemic control. Diabetes Care 32:1119-1131

16. Bogun M, Inzucchi SE (2013) Inpatient management of diabetes and hyperglycemia. Clin Ther 35:724-733

17. Van den Berghe G, Wouters P, Weekers F et al (2001) Intensive insulin therapy in critically ill patients. NEngl J Med 345:1359-1367

18. Finfer S, Chittock DR, Su SY et al (2009) NICESUGAR Study Investigators. Intensive versus conventional glucose control in critically ill patients. NEngl J Med 360:1283-1297

19. Kansagara D, Fu R, Freeman M, Wolf F, Helfand M (2011) Intensive insulin therapy in hospitalized patients: a systematic review. Ann Intern Med 154:268-282

20. Sathya B, Davis R, Taveira T, Whitlatch H, Wu WC (2013) Intensity of peri-operative glycemic control and postoperative outcomes in patients with diabetes: a meta-analysis. Diabetes Res Clin Pract 102:8-15

21. Umpierrez G, Cardona S, Pasquel F et al (2015) Randomized controlled trial of intensive versus conservative glucose control in patients undergoing coronary artery bypass graft surgery: GLUCO-CABG trial. Diabetes Care 38:1665-1672

22. Duncan $A E$, Abd-Elsayed A, Maheshwari $A, X u M$, Soltesz E, Koch CG (2010) Role of intraoperative and postoperative blood glucose concentrations in predicting outcomes after cardiac surgery. Anesthesiology 112:860-871

23. Furnary AP, Wu Y, Bookin SO (2004) Effect of hyperglycemia and continuous intravenous insulin infusions on outcomes of cardiac surgical procedures: the Portland Diabetic Project. Endocr Pract 10(Suppl. 2):21-33

24. Wang CCL, Draznin B (2013) Practical approach to management inpatient hyperglycemia in selected populations. Hosp Pract 41:45-53

25. Christensen MB, Gotfredsen A, Nørgaard K (2017) Efficacy of basal-bolus insulin regimens in the inpatient management of non-critically ill patients with type 2 diabetes: a systematic review and meta-analysis. Diabetes Metab Res Rev 33:e2885
26. Lee $Y-Y$, Lin Y-M, Leu W-J et al (2015) Slidingscale insulin used for blood glucose control: a meta-analysis of randomized controlled trials. Metabolism 64:1183-1192

27. Kitabchi $A E$, Umpierrez GE, Miles JM, Fisher JN (2009) Hyperglycemic crises in adult patients with diabetes. Diabetes Care 32:1335-1343

28. Cobaugh DJ, Maynard G, Cooper L et al (2013) Enhancing insulin-use safety in hospitals: practical recommendations from an ASHP Foundation expert consensus panel. Am J Health Syst Pharm 70:1404-1413

29. Rice MJ, Coursin DB (2016) Glucose meters: here today, gone tomorrow? Crit Care Med 44:e97-e100

30. Rice MJ, Smith JL, Coursin DB (2017) Glucose measurement in the ICU: regulatory intersects reality. Crit Care Med 45:741-743

31. Akirov A, Grossman A, Shochat T, Shimon I (2017) Mortality among hospitalized patients with hypoglycemia: insulin related and noninsulin related. JClin Endocrinol Metab 102:416-424

32. Amori RE, Pittas AG, Siegel RD et al (2008) Inpatient medical errors involving glucoselowering medications and their impact on patients: review of 2,598 incidents from a voluntary electronic error-reporting database. Endocr Pract 14:535-542

33. Alwan D, Chipps E, Yen P-Y, Dungan K (2017) Evaluation of the timing and coordination of prandial insulin administration in the hospital. Diabetes Res Clin Pract 131:18-32

34. Hung AM, Siew ED, Wilson OD et al (2018) Risk of hypoglycemia following hospital discharge in patients with diabetes and acute kidney injury. Diabetes Care 41:503-512

35. Umpierrez G, Korytkowski M (2016) Diabetic emergencies-ketoacidosis, hyperglycaemic hyperosmolar state and hypoglycaemia. Nat Rev Endocrinol 12:222-232

36. Mabrey ME, Setji TL (2015) Patient self-management of diabetes care in the inpatient setting: pro. JDiabetes Sci Technol 9:1152-1154

37. Shah AD, Rushakoff RJ (2015) Patient selfmanagement of diabetes care in the inpatient setting: con. J Diabetes Sci Technol 9:1155-1157

38. Umpierrez GE, Klonoff DC (2018) Diabetes technology update:use of insulin pumps and continuous glucose monitoring in the hospital. Diabetes Care 41:1579-1589

39. Houlden RL, Moore S (2014) In-hospital management of adults using insulin pump therapy. Can J Diabetes 38:126-133

40. Pichardo-Lowden AR, Fan CY, Gabbay RA (2011) Management of hyperglycemia in the nonintensive care patient: featuring subcutaneous insulin protocols. Endocr Pract 17:249-260

41. Roberts A, James J, Dhatariya K (2018) Joint British Diabetes Societies (JBDS) for Inpatient Care. Management of hyperglycaemia and steroid (glucocorticoid) therapy: a guideline from the Joint British Diabetes Societies (JBDS) for Inpatient Care group. Diabet Med 35:1011-1017

42. Smiley DD, Umpierrez GE (2006) Perioperative glucose control in the diabetic or nondiabetic patient. South Med J 99:580-589 (quiz 590-591)

43. Buchleitner AM, Martínez-Alonso M, Hernández M, Solà I, Mauricio D (2012) Perioperative glycaemic control for diabetic patients undergoing surgery. Cochrane Database Syst Rev. https://doi.org/10. 1002/14651858.CD007315.pub2

44. Gianchandani R, Dubois E, Alexanian S, RushakoffR (2016) Preoperative, intraoperative, and postoperative glucose management. In: Draznin B (Hrsg) Managing diabetes and hyperglycemia in the 
hospital setting. American Diabetes Association, Alexandria, S129-144

45. Chiang HY, Lin K-TR, Hsiao Y-A, Huang H-C, Chang S-N, Hung C-H, Chang Y, Wang Y-C, Kuo C-C (2020) Association between preoperative blood glucose level and hospital length of stay for patients undergoing appendectomy or laparoscopic cholecystectomy. Diabetes Care 44:107-115

46. Noordzij PG, Boersma $E$, Schreiner $F$ et al (2007) Increased preoperative glucose levels are associated with perioperative mortality in patients undergoing noncardiac, nonvascular surgery. Eur J Endocrinol 156:137-142

47. Hatzakorzian R, BuiH,Carvalho G, Shan WL, SidhuS, Schricker T (2011) Fasting blood glucose levels in patients presenting for elective surgery. Nutrition 27:298-301

48. AbdelmalakBB, KnittelJ, AbdelmalakJB etal (2014) Preoperative blood glucose concentrations and postoperative outcomes after elective non-cardiac surgery: an observational study. $\mathrm{Br} J$ Anaesth 112:79-88

49. Wang R, Panizales MT, Hudson MS, Rogers SO, Schnipper JL (2014) Preoperative glucose as a screening tool in patients without diabetes. J Surg Res 186:371-378

50. Bock M, Johansson T, Fritsch G et al (2015) The impact of preoperative testing for blood glucose concentration and haemoglobin A1 c on mortality, changes in management and complications in noncardiac elective surgery: a systematic review. Eur J Anaesthesiol 32:152-159

51. De Hert S, Staender S, Fritsch G et al (2018) Preoperative evaluation of adults undergoing elective noncardiac surgery: updated guideline from the European Society of Anaesthesiology. Eur J Anaesthesiol 35:407-465

52. Rajendran R, Kerry C, Rayman G, on behalf of the MaGIC Study Group (2014) Temporal patterns of hypoglycaemia and burden of sulfonylurea-related hypoglycaemia in UK hospitals: a retrospective multicentre audit of hospitalised patients with diabetes. BMJOpen 4:e5165

53. Montejano L, Vo L, McMorrow D (2016) Transitions of care for people with type 2 diabetes: utilization of antihyperglycemic agents pre- and posthospitalization. Diabetes Ther 7:91-103

54. Pasquel FJ, Klein R, Adigweme A et al (2015) Metformin-associated lactic acidosis. Am J Med Sci 349:263-267

55. Vellanki P, Rasouli N, Baldwin D et al (2019) Glycaemic efficacy and safety of linagliptin compared to basal-bolus insulin regimen in patients with type 2 diabetes undergoing noncardiac surgery: a multicentre randomized clinical trial. Diabetes Obes Metab 21:837-843

56. Garg R, Schuman B, Hurwitz S, Metzger C, Bhandari $S$ (2017) Safety and efficacy of saxagliptin forglycemic control in non-criticallyill hospitalized patients. BMJ Open Diabetes Res Care 5:e394

57. Nyström T, Gutniak MK, Zhang Q et al (2004) Effects of glucagon-like peptide-1 on endothelial function in type 2 diabetes patients with stable coronary artery disease. Am J Physiol Endocrinol Metab 287:E1209-E1215

58. Sokos GG, Bolukoglu H, German Jetal (2007) Effect of glucagon-like peptide-1 (GLP-1) on glycemic control and left ventricular function in patients undergoing coronary artery bypass grafting. Am J Cardiol 100:824-829

\section{Importance of blood glucose values in the hospital}

Background: Hyperglycemia in people with and without diabetes who are hospitalized is associated with a substantial increase in morbidity, mortality, and health care costs. During hospitalization, metabolic decompensation frequently occurs as a result of various events or adjunctive treatment. Due to the increased risk for an increase in morbidity associated with longer hospital stay as well as higher costs and mortality, a close look at the significance of glucose levels and the forms of therapy in the hospital is warranted.

Materials and methods: Current findings, reviews, and basic data were analyzed and the results are summarized and discussed in a brief review.

Conclusion: Persistent hyperglycemia in the hospital is common and often associated with poor hospital outcomes. Continuous insulin infusion remains the therapy of choice during hyperglycemic crises and critical illness. In noncritically ill patients, insulin also remains the treatment of choice in patients with marked hyperglycemia, high insulin doses already known in outpatients, type 1 diabetes, or patients with steroid-induced hyperglycemia.

\section{Keywords}

Diabetes mellitus · Hyperglycemia $\cdot$ Hypoglycemia $\cdot$ Insulin $\cdot$ Hospitalization 